\title{
A review of research on flower-colored mutants of fragrant cyclamens induced by ion-beam irradiation
}

\begin{abstract}
Ornamental plants are extremely valuable in terms of horticulture and are expected to be in high demand in the future. However, molecular analysis of ornamental plants is very difficult because of the complexity of their genetic backgrounds. To solve this problem, comparative analysis using mutations is a simple and effective approach. In this review, we summarize current molecular research on flower coloration in fragrant wild cyclamen, describing the fragrant cultivars (Cyclamen persicum $\times C$ purpurascens) and their flower-colored mutants induced by ion-beam irradiation. These mutants are not only valuable horticultural, but are also useful for molecular analyses of flower coloration in ornamental plants.
\end{abstract}

Keywords: anthocyanin, cyclamen, flavonoid, flower color, ion-beam breeding

\author{
Volume I Issue 3 - 2017 \\ Yusuke Akita, ${ }^{1,2}$ Shiho Morimura,' Parisa \\ Loetratsami, ${ }^{2}$ Hiroshi Ishizaka ${ }^{3}$ \\ 'Department of Applied Chemistry, Saitama Institute of \\ Technology, Japan \\ 2Department of Life Science and Green Chemistry, Saitama \\ Institute of Technology, Japan \\ ${ }^{3}$ Saitama Agricultural Technology Research Center, Japan
}

\begin{abstract}
Correspondence: Yusuke Akita, Saitama Institute of Technology, 1690 Fusaiji Fukaya, Saitama, JPN 369-0293, Japan, Tel +8I-48-585-6893, Email akita@sit.ac.jp
\end{abstract}

Received: November 17, 2017 | Published: December 19, 2017

\section{Introduction}

Of the important traits that determine the overall value of a flower, color is perhaps the most important. In many flowers, the main component of flower color is anthocyanin, a type of flavonoid. ${ }^{1}$ Numerous genes involved in anthocyanin biosynthesis have been analyzed in various plants, and their biosynthesis pathways determined. ${ }^{2,3}$ In addition to anthocyanins, flavonols and organic acids also play a role in flower color as 'co-pigments' ${ }^{4}$ Fragrant cyclamen cultivars were previously created using embryo rescue and chromosome doubling to perform interspecific hybridization between Cyclamen purpurascens as the pollen parent and cultivars of $C$. persicum. ${ }^{5}$ Initially, the fragrant cyclamen series consisted of purple or pink flowers only and increased variations in flower color were desired. Our overall aim, therefore, is to identify the genes related to cyclamen flower color with the goal of creating different flowercolor mutants by using ion-beam irradiation. Here, we summarize the results so far and consider future development of this research The main component of cyclamen flower color is anthocyanin, with certain flavonols also involved in co-pigmentation. ${ }^{6}$ Approximately 20 homologues of flavonoid biosynthesis-related genes have so far been isolated from $C$. purpurascens and expression patterns examined by RT-PCR. ${ }^{7}$ As a result, expression of a number of genes was confirmed in the slip and eye, with lower expression in the leaves. In addition, two flavonol synthase genes (CpurFLS1 and CpurFLS2) were also isolated and detailed analyses performed. As a result, CpurFLS2 is thought to play a role in flower color. ${ }^{8}$ However, further clarification of direct involvement in flower color is required. For example, 3-O-glucosyltransferase (3GT) not only target anthocyanidin, but is also thought to act as a glycosidase of other flavonoids in freesia. ${ }^{9}$ In the future, clarification of the enzyme genes directly related to flower color is therefore required via comparative analysis using flowercolored mutants and identification of the mutant factors.

Ion-beam irradiation technology has been used to create flowercolored mutants of fragrant cyclamen. ${ }^{10}$ To do so, ion-beams are used as the physical mutagen rather than gamma rays, x-rays, or neutrons, which are predominantly used for mutation induction in plants. ${ }^{11,12}$ We previously carried out 'targeted mutation breeding' of flower color using ion-beam irradiation and genomic information, ${ }^{13}$ and from the original cultivar 'Koko-no-kaori' (KO), which has strong reddish-purple (Royal Horticultural Society Colour Chart; RHS No: N78B)flowers, were able to produce white- (KOpw) and strong purplish-red(RHS No: 71B) flower (KOrp) colors (Figure 1A). Using another cultivar, 'Kaori-no-mai' (KM), which has strong reddish-purple (RHS No: NN78B) flowers, two variants which have deep purplish-red (RHS No: 71A) color in their flower, KMDp and 'Miyabi-no-mai' (MY) were created (Figure 1B). While using a third cultivar 'Uruwashi-no-kaori' (UR) which has light purple (RHS No: 75B) flower-color, 'Tennyo-no-mai' (TN), which has vivid purplishred (RHS No: 67B) flowers, was created (Figure 1C). Furthermore, we recently created new flower-colored variant from KM (DRP, (Figure 1B). Its flower color is not suit to any colors on RHS Colour Chart (most nearest to RHS No: 59A). Subsequent analysis of flower color components using high-performance liquid chromatography (HPLC) confirmed that the anthocyanin malvidin 3,5-diglucoside (Mv3,5dG) is not present in KOpw. ${ }^{14}$ In contrast, Mv3,5dG was found to be the main flower color component in KOrp, while the presence of flavonols could not be confirmed. ${ }^{14}$ These findings are likely due to mutations in the genes involved in flavonoid biosynthesis. For example, KOrp is thought to harbor a mutation of the CpurFLS2-like gene from C. purpurascens, and therefore, comparative analysis is currently underway using KO and KOrp. Furthermore, in KMDp, mutation of Mv3,5dG to delphinidin 3,5-diglucoside (Dp3,5dG) was confirmed. ${ }^{15}$ Changes were also observed in MY from Mv3,5G to malvidin 3-glucoside (Mv3dG). ${ }^{14}$ In contrast, no significant changes to the structure or component ratio of anthocyanins were observed in $\mathrm{TN}$; rather, the content of anthocyanin increased greatly, while that of flavonols decreased. ${ }^{14}$ These findings suggest that rather than a mutation, flavonoid biosynthesis was activated; or perhaps the negative regulator of the enzyme gene group involved in flavonoid biosynthesis was mutated.

The dominant feature of flower-color mutants created by ionbeam irradiation is their clear genetic background. This is a very important in terms of molecular biological research of horticultural 
plants with a long cultivation history. For example, KMDp is thought to harbor a mutant of methyltransferase (MT), which functions as an anthocyanin. We therefore isolated four MT genes as candidate mutant factors. Of these, CkmOMT2 was found to function in methylation of delphinidin-derived anthocyanin, playing a direct role in flower color. ${ }^{16}$ This finding could then be easily confirmed thanks to the clear genetic background of KMDp. In the future, our aim is to carry out further comparative analysis using additional ion-beaminduced flower-color mutants in order to confirm the factors involved in flower coloration.
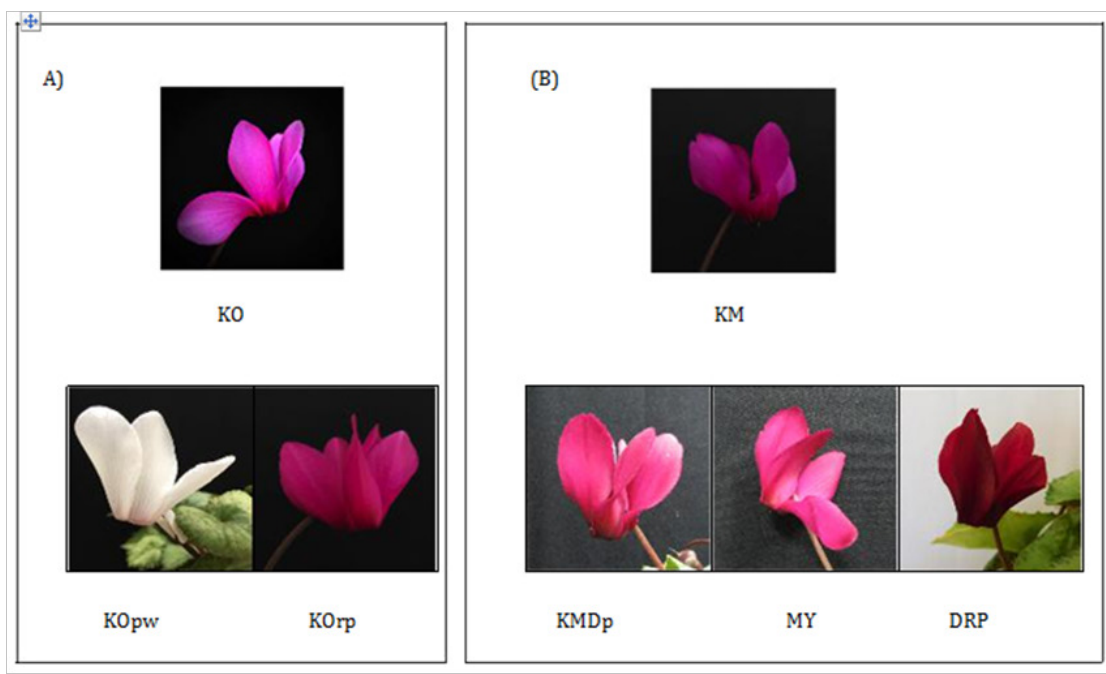

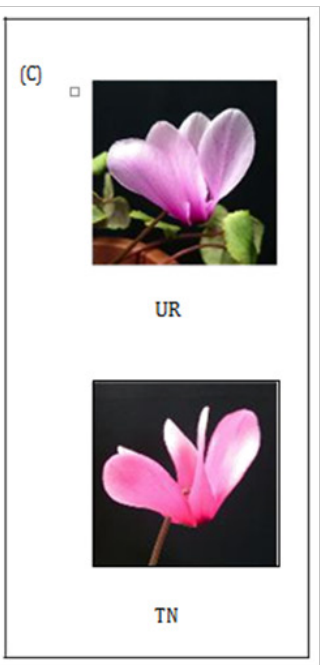

Figure I: Flowers of fragrant cyclamens. 'Koko-no-kaori' $(\mathrm{KO})$ and its mutants.

\section{Acknowledgments}

None.

\section{Conflict of interest}

The author declares no conflict of interest.

\section{References}

1. Harborne JB, Williams CA. Advances in flavonoid research since 1992. Phytochemistry. 2000;55(6):481-504.

2. Grotewold E. The genetics and biochemistry of foral pigments. Annu Rev Plant Biol. 2006;57:761-780.

3. Forkmann G. Flavonoids as Flower pigments: The formation of the natural spectrum and its extension by genetic engineering. Plant Breeding. 1991;106:1-26.

4. Yazaki Y. Co-pigmentation and the color change with age in petals of Fuchsia hybrida. Botanical Magazine. 1976;89(1):45-57.

5. Ishizaka H. Interspecific hybridization by embryo rescue in the genus Cyclamen. Plant Biotechnology. 2008;25(6):511-519.

6. Takamura T, Aizawa M, Kim SY, et al. Inheritance of flower color pigment in crosses between cyclamen cultivars and Cyclamen purpurascens. Acta Horticulture. 2005;673:437-441.

7. Hase Y, Akita Y, Kitamura S, et al. Development of an efficient mutagenesis technique using ion beams: Toward more controlled mutation breeding. Plant Biotechnology. 2012;29(3):193-200.

8. Akita Y, Kitamura S, Mikami R, et al. Identification of functional flavonol synthase genes from fragrant wild cyclamen (Cyclamen purpurascens). Journal of Plant Biochemistry and Biotechnology. 2018;27(2):147-155.
9. Sun W, Liang L, Meng X, et al. Biochemical and molecular characterization of a flavonoid 3-O-glycosyltransferase responsible for anthocyanins and flavonols biosynthesis in Freesia hybrida. Front Plant Sci. 2016;7:410.

10. Ishizaka H, Kondo E, Kameari N. Production of novel flower color mutants from the fragrant cyclamen (Cyclamen persicum $\mathrm{x}$ C. purpurascens) by ion-beam irradiation. Plant Biotechnology. 2012;29:201-208.

11. Yamaguchi H, Nagatomi S, Morishita T, et al. Mutation induced with ion-beam irradiation in rose. Nuclear Instruments and Methods in Physics Research Section B. 2003;206:561-564.

12. Watanabe H. Significance and expectations of ion beam breeding. Gamma field Symposia. 2001;40:15-19.

13. Tanaka A. Targeted mutation breeding of flower color by taking the advantage of ion-beam irradiation and genomic information. Plant Biotechnology. 2012;29(3):191-192.

14. Nakayama M, Tanikawa N, Morita Y, et al. Comprehensive analyses of anthocyanin and related compounds to understand flower color change in ion-beam mutants of cyclamen (Cyclamen spp.) and carnation (Dianthus caryophyllus). Plant Biotechnology. 2012;29(3):215-221.

15. Kondo E, Nakayama M, Kameari N, et al. Red-purple flower due to delphinidin 3,5-diglucoside, a novel pigment for Cyclamen spp., generated by ion-beam irradiation. Plant Biotechnology. 2009;26(5):565-569.

16. Akita Y, Kitamura S, Hase Y, et al. Isolation and characterization of the fragrant cyclamen $O$-methyl transferase involved in flower coloration. Planta. 2011;234(6):1127-1136. 